\title{
Value of spiral CT multi-parameter combined preoperative evaluation of microvascular invasion in small liver cancer
}

\author{
Kun $\mathrm{Li}^{1}$, Yongjun Peng ${ }^{2}$, \\ Hongzhe Tian ${ }^{3}$, Hailin He${ }^{4}$
}

\section{ABSTRACT}

Objective: To explore the value of multi-slice spiral CT (MSCT) in predicting microvascular invasion in hepatocellular carcinoma (HCC).

Methods: The CT and clinical data of 102 patients with $\mathrm{HCC}$ were collected for retrospective analysis from January 2018 to December 2020 at Baoji Center Hospital, China. They were divided into two groups based on the pathological results with or without microvascular invasion. The independent sample t-test was used to compare the age, alpha-fetoprotein (AFP) value, tumor size, and tumor enhancement of the two groups. CT value; $x 2$ test was used to compare gender, hepatitis type, liver function classification, degree of classification, degree of tumor smoothness, envelope, peripheral enhancement, etc. between the two groups.

Results: There were 52 cases of non-microvascular invasion and 50 cases of microvascular invasion. The tumor size, grade, degree of margin, capsule, portal vein CT value, and peripheral enhancement were related to microvascular invasion.

Conclusion: Microvascular invasion of HCC can be predicted by MSCT manifestations before surgery.

KEYWORDS: Multi-slice spiral CT, Preoperative, Microvascular invasion, Small liver cancer, Hepatocellular carcinoma.

doi: https://doi.org/10.12669/pjms.37.6-WIT.4851

How to cite this:

Li K, Peng Y, Tian H, He H. Value of spiral CT multi-parameter combined preoperative evaluation of microvascular invasion in small liver cancer. Pak J Med Sci. 2021;37(6):1605-1609. doi: https://doi.org/10.12669/pjms.37.6-WIT.4851

This is an Open Access article distributed under the terms of the Creative Commons Attribution License (http://creativecommons.org/licenses/by/3.0), which permits unrestricted use, distribution, and reproduction in any medium, provided the original work is properly cited.

1. Kun Li,

Associate Chief Physician.

2. Yongjun Peng, Chief Physician.

Department of Radiology,

Zhuhai People's Hospital, Zhuhai 519000,

Guangdong, China.

3. Hongzhe Tian,

Associate Chief Physician, Master of Medicine,

4. Hailin $\mathrm{He}$,

Associate Chief Physician.

1,3,4: Department of Medical Imaging,

Baoji Center Hospital, Baoji 721008 ,

Shaanxi, China.

Correspondence:

Hailin He,

Department of Medical Imaging,

Baoji Center Hospital,

Baoji 721008, Shaanxi, China.

Email: hehailinhaanxi@yeah.net

* Received for Publication:

* Revision Received:

* Final Revision Accepted:
June 8, 2021

June 12, 2021

July 10, 2021

\section{INTRODUCTION}

Many researchers have tried to evaluate microvascular invasion using methods such as hepatic angiography and enhanced MRI. However, the imaging manifestations of microvascular invasion of HCC are not clear. This study aims to predict the microvascular invasion of HCC by multi-slice spiral CT (MSCT) imaging. ${ }^{1-3}$ At this stage, lung CT imaging is still the primary method for diagnosing lung cancer in China. However, when tuberculosis and lung cancer coexist, lung cancer diagnosis and treatment are easily delayed due to similar symptoms. Therefore, the lung CT imaging of the two is identified. The characteristics are of great significance for guiding the clinical diagnosis of tuberculosis with lung cancer. 


\section{METHODS}

Retrospective analysis of the image data of all patients who underwent radical resection of HCC in the Baoji Center Hospital, from January 2018 to December 2020 was performed after taking IRB approval on March 24, 2021.

Inclusion criteria: (1) no macrovascular tumor thrombosis was found in all image data There is only one isolated mass; (2) The interval between CT examination and surgery should be less than 1 month. Finally, 102 patients with HCC were selected as the research subjects, 76 males and 26 females, aged 37 to 85 years, with an average age of 61.8 years, 55 patients with hepatitis $B, 36$ patients with hepatitis $C$, four patients with hepatitis $B$ and $\mathrm{C}$, seven cases of alcoholic hepatitis. Child-Pugh classification of liver function: 98 cases of grade A, three cases of grade B, one case of grade C. ${ }^{4,5}$

CT examination. The TOSHIBA Aquilion 64-slice spiral CT scan was used. The scanning layer thickness was $5 \mathrm{~mm}$, the pitch was 0.75 , the tube voltage was $120 \mathrm{kV}$, and the tube current was 180 $220 \mathrm{mAs}$. The whole liver was scanned first and then the scan was enhanced. It was $1.5 \mathrm{ml} / \mathrm{kg}$ body weight. It was injected through a superficial vein of the elbow with a double-barreled high-

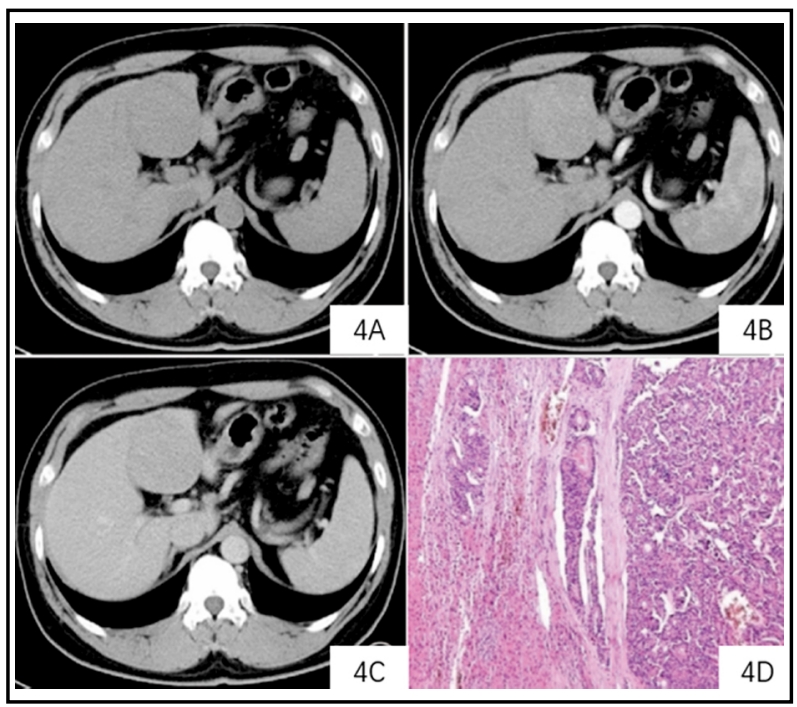

Fig.1: CT scan of the left lobe of the liver shows a circular low-density lesion.

Note: A 51-year-old man with a CT scan of the left lobe of the liver showed a circular low-density lesion of approximately $5.8 \mathrm{~cm} \times 5.4 \mathrm{~cm}$, with a CT value of approximately $45 \mathrm{HU}(4 \mathrm{~A})$; uneven enhancement of arterial lesions, and a CT value of $69 \mathrm{HU}(4 \mathrm{~B})$; The CT value of portal vein $(4 \mathrm{C})$ was $81 \mathrm{HU}$. Pathology (HE staining $x$ 200) showing enveloped vascular cancer thrombus (4D). pressure syringe at a flow rate of about $3 \mathrm{ml} / \mathrm{s}$. The arterial phase, portal vein phase, and delayed phase scan were performed at 30s, 60s, and 120s, respectively.

Imaging Analysis. Two deputy chief physicians observed CT images on the workstations and gave a diagnosis. The tumor size, margins, capsules, enhancement methods, and peripheral enhancement were evaluated. When inconsistencies were negotiated with each other. Smooth tumor edges appear as smooth arcs on imaging images in all directions. The envelope is a ring-shaped high-density shadow with a clear edge of the tumor at the portal vein or delayed stage. The envelope surrounding the tumor $\geq 40 \%$ is recorded as an envelope; $<40 \%$ is recorded as an envelope-free. When measuring CT values, avoid areas such as hemorrhage, necrosis, and cystic changes. Take three measurements and take the average value. Select the same level for each phase of the same lesion. The halo sign needs to be observed around the tumor enhancement. The halo sign is a ring-shaped or band-like enhancement of the liver parenchyma around the tumor at the late stage of arteries or early portal veins, and the delay period decreases with equal density. ${ }^{6}$

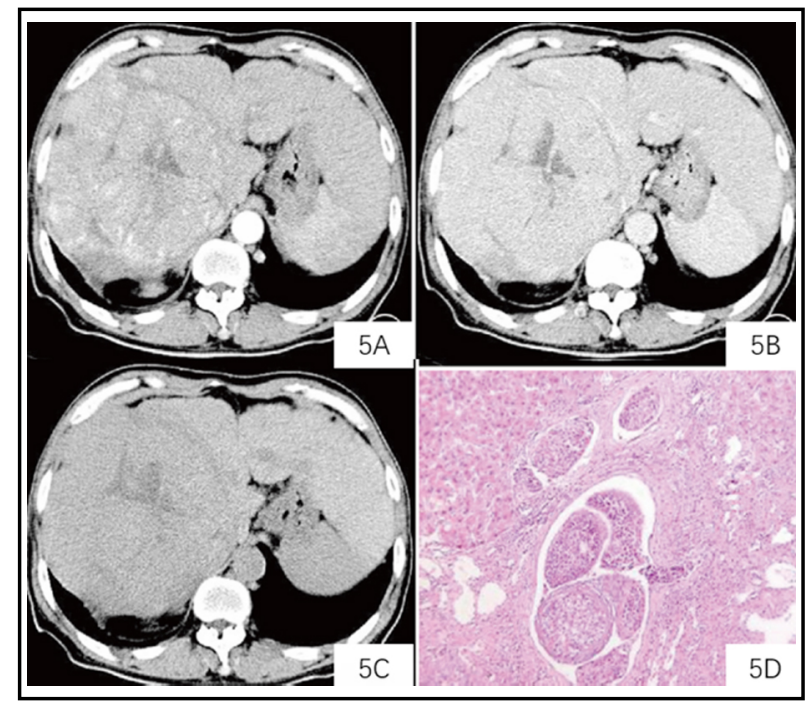

Fig.2: A plain scan of the right lobe of the liver shows a low-density mass

Note: The patient was male, 58 years old. A plain scan of the right lobe of the liver revealed a low-density mass of $10.1 \mathrm{~cm} \times 11.5 \mathrm{~cm}$. The CT value was approximately $49 \mathrm{HU}(5 \mathrm{~A})$. The solid components in the arterial phase were significantly enhanced, and the $\mathrm{CH}$ value was approximately $91 \mathrm{HU}(5 \mathrm{~B})$. The $\mathrm{CT}$ value in the portal vein phase $(5 \mathrm{C})$ was $78 \mathrm{HU}$. Pathology (HE staining $\times$ $200)$ showed tumor thrombus in the small vein $(5 E)$. 
Pathological analysis. The pathologist observed all the postoperative pathological sections and found that tumor thrombus aggregated in the small blood vessels (central vein, portal vein branch and capsular venule), or cancer cells appeared in the vascular endothelial layer and smooth muscle layer, then it was determined as HCC. Microvascular invasion. According to Edmondson Steiner classification (grade 1 to 5), the degree of tumor differentiation was determined.

Statistical analysis. SPSS 18. 0 statistical software was used to analyze. Micro-vascular invasion was divided into two groups according to pathological results. Independent sample $t$ test was used to compare the age, alpha-fetoprotein (AFP) value, tumor size, and tumor-enhanced CT value of the two groups of patients. $\chi 2$ test was used to compare the sex, hepatitis type, liver function grade, tumor grade, tumor smoothness, envelope, and halo sign between the two groups. The difference was statistically significant with $\mathrm{P}<0.05 .{ }^{7,8}$

\section{RESULTS}

According to the pathological results, they were divided into a non-microvascular invasion group (52 cases) and a microvascular invasion group (50 cases). The clinical and imaging data of the two groups of patients are compared in Table-I and Fig. 1 and 2. There was no significant difference between the two groups in terms of age, gender, hepatitis type, liver function classification, AFP value, arterial phase and delayed phase CT value (P> 0.05); in tumor pathological classification, size, margin, envelope. There was a statistically significant difference in $\mathrm{CT}$ value and peripheral enhancement in portal vein stage $(\mathrm{P}<0.05)$.

Table-I: Relationship between clinical and imaging data and microvascular invasion.

\begin{tabular}{|c|c|c|c|c|}
\hline Clinical and imaging data & $\begin{array}{l}\text { Non-microvascular } \\
\text { invasion group }\end{array}$ & $\begin{array}{l}\text { Microvascular } \\
\text { invasion group }\end{array}$ & $\begin{array}{l}t \text {-value or } \\
x^{2} \text { value }\end{array}$ & P-value \\
\hline Age & $63.31 \pm 5.02$ & $65.96 \pm 5.80$ & 2.473 & 0.362 \\
\hline Male (example) & 37 & 39 & 0.629 & 0.428 \\
\hline Hepatitis B (example) & 28 & 27 & 0.000 & 0.988 \\
\hline Hepatitis C (example) & 19 & 17 & 0.072 & 0.789 \\
\hline Hepatitis B + C (Example) & 2 & 2 & - & 1 \\
\hline Alcoholic hepatitis (example) & 3 & 4 & - & 0.713 \\
\hline \multicolumn{5}{|l|}{ Liver function classification } \\
\hline A (example) & 51 & 47 & - & 0.358 \\
\hline B (example) & 1 & 2 & - & 0.614 \\
\hline $\operatorname{AFP}(\mu \mathrm{g} / \mathrm{mL})$ & $416.29 \pm 576.31$ & $337.84 \pm 529.07$ & 0.715 & 0.476 \\
\hline \multicolumn{5}{|l|}{ Edmondson Steiner grading } \\
\hline 1/2 level (example) & $7 / 25$ & $2 / 18$ & 4.732 & 0.030 \\
\hline Level 3/4 (example) & $14 / 6$ & $20 / 10$ & - & - \\
\hline Tumor size $(\mathrm{cm})$ & $3.67 \pm 0.83$ & $4.40 \pm 1.32$ & 3.218 & 0.002 \\
\hline $\begin{array}{l}\text { Tumor edges are not smooth } \\
\text { (example) }\end{array}$ & 19 & 35 & 11.456 & 0.001 \\
\hline Tumor has an envelope (example) & 39 & 26 & 5.833 & 0.016 \\
\hline Arterial CT value $(\mathrm{HU})$ & $79.88 \pm 11.78$ & $75.94 \pm 9.39$ & 1.866 & 0.065 \\
\hline $\mathrm{CT}$ value of portal vein $(\mathrm{HU})$ & $70.62 \pm 10.96$ & $84.80 \pm 12.50$ & 6.102 & $<0.001$ \\
\hline Delay CT value (HU) & $69.17 \pm 12.70$ & $72.04 \pm 9.50$ & 1.29 & 0.201 \\
\hline Halo sign (example) & 29 & 38 & 4.629 & 0.031 \\
\hline
\end{tabular}




\section{DISCUSSION}

The results of this study show that the CT value of arterial tumors in the non-microvascular invasion group is slightly higher than that in the microvascular invasion group; the $\mathrm{CT}$ value of portal vein tumors in the non-microvascular invasion group is significantly lower than that in the microvascular invasion group. It showed that the portal vein phase of the tumor in the microvascular invasion group continued to strengthen, and the $\mathrm{CT}$ value continued to increase. The enhancement method was the continuous enhanced type in the portal vein phase. The CT value was significantly higher than that in the non-microvascular invasion group. The blood supply of the tumor was closely related to the enhancement method.

The enhancement method suggests that the tumor is dually supplied by the hepatic artery and the portal vein. After microvascular invasion, the tumor will accelerate the release of tumor angiogenesis promoting factors, such as hypoxiainducible factor-1a (HIF-1a) and vascular endothelial growth factor (VEGF). New blood vessels will diversify the blood supply to the tumor. The tumor enhancement method reflects the characteristics of blood supply. When the hepatic artery and portal vein are dual-supply, the arterial phase and the portal vein phase are gradually strengthened, and the strengthening duration is longer. 9,10 Microvascular invasion tumors continuously release a large amount of HIF-1a and VEGF, and induce many micro angiogenesis. The enhancement of the arterial phase and the portal vein phase is mainly affected by angiogenesis. The delayed phase enhancement is mainly related to the extravascular space and the permeability of blood vessels. These factors are related to HIF-1a and VEGF, which lay the pathophysiology of tumor enhancement. Therefore, HCC microvascular invasion can be predicted by the enhancement method of tumor portal vein phase and CT value increase. The non-smooth tumor edge has implications for predicting microvascular invasion. ${ }^{11,12}$ It is believed that solitary masses with nodular protrusions and multiple nodular fusion are more likely to be accompanied by microvascular invasion than a single mass. However, preoperative CT imaging is difficult to distinguish between multiple nodules and solitary masses with nodule protrusions. ${ }^{13,14}$ The results of this study showed that intra-tumoral arteries were statistically significant in predicting microvascular invasion. The intra-tumoral artery sign was more likely to appear in the microvascular invasion group than in the nonmicrovascular invasion group. It was in line with the study of Segal ${ }^{15}$, which proved that this sign was related to angiogenesis and cell proliferation. The peri-tumor enhancement, low-density halo sign, and tumor-to-liver difference were found to be not statistically significant in predicting HCC microvascular invasion. Some studies have used enhancement around the tumor, lowdensity halo sign, and tumor-liver difference as parameters, suggesting an increased risk of tumor microvascular invasion. This may be due to the different imaging methods and the low percentage of surrounding tumor enhancement on dynamic CT images. The results still need to be confirmed using an expanded sample size. At the same time, the presence of tumor envelope and microvascular invasion did not show a significant correlation. However, studies have found that, the fibrous envelope in HCC is a favorable prognostic factor because the envelope can prevent liver cancer from invading the adjacent liver parenchyma. However, Adachi ${ }^{16}$ showed that, fibrous enveloped blood vessels were often invaded by cancer cells, and pointed out that the presence of fibrous envelope was a predictor of portal invasion. The relationship between the existence of tumor capsule and the occurrence of MVI still remains academically controversial, and further research is needed. ${ }^{17,18}$

\section{CONCLUSIONS}

In short, the size of HCC tumors, Edmondson Steiner classification, marginal, envelope, portal vein enhancement characteristics, peripheral enhancement are closely related to microvascular invasion. When the tumor is larger, Edmondson Steiner classification is higher, matte edges, no envelope, portal vein Continued intensification, enhanced CT values and peripheral intensification are often suggestive of microvascular invasion when there are halo signs. Patients need more active treatment, expanding the scope of surgery or combining with adjuvant treatment to reduce the recurrence rate. 


\section{REFERENCES}

1. Cong WM, Bu H, Chen J, Dong H. Guideline committee. practice guidelines for the pathological diagnosis of primary liver cancer: 2015 update. World J Gastroenterol. 2016;22(42):9279. doi: 10.3748/wjg.v22.i42.9279

2. Zhang Y, Li B, Shi, H, Yu H Gu Y, Xiu Y. Added value of SPECT / spiral CT versus SPECT or CT alone in diagnosing solitary skeletal lesions. Nuklearmedizin. 2017;56(4):139145. doi: 10.3413/Nukmed-0886-17-03

3. Li L, Li B, Zhang M, Hbv dna levels impact the prognosis of hepatocellular carcinoma patients with microvascular invasion. Medicine (Baltimore). 2019;98(27):e16308. doi: 10.1097/MD.0000000000016308

4. Orcutt ST, Anaya DA. Liver resection and surgical strategies for management of primary liver cancer. Cancer Control. 2018;25(1):1073274817744621. doi: $10.1177 / 1073274817744621$

5. He NN, van Iperen L, de Jong D, Szuhai K, Helmerhorst FM, van der Westerlaken LAJ. Human extravillous trophoblasts penetrate decidual veins and lymphatics before remodeling spiral arteries during early pregnancy. Plos One. 2017;12(1):e0169849. doi: 10.1371/journal.pone.0169849

6. Li MX, Jin ZX, Zhou JG, Ying JM, Zhao H. Prognostic value of lymph node ratio in patients receiving combined surgical resection for gastric cancer liver metastasis: results from two national centers in china. Medicine (Baltimore). 2016;95(16):e3395. doi: 10.1097/MD.0000000000003395

7. Erstad DJ, Tanabe KK. Prognostic and therapeutic implications of microvascular invasion in hepatocellular carcinoma. Ann Surg Oncol. 2019;26(5):1474-1493. doi: 10.1245/s10434-019-07227-9

8. Wei XB, Li N, Li SS, Shi J, Cheng SQ. Hepatitis b virus infection and active replication promote the formation of vascular invasion in hepatocellular carcinoma. BMC Cancer. 2017;17(1):304. doi: 10.1186/s12885-017-3293-6

9. Ling CQ, Fan J, Lin HS, Shen F, Xu ZY, Lin LZ, et al. Chinese Integrative Therapy of Primary Liver Cancer Working Group. Clinical practice guidelines for the treatment of primary liver cancer with integrative traditional Chinese and Western medicine. J Integr Med. 2018;16(4):236-248. doi: 10.1016/j.joim.2018.05.002

10. Jurčić, P, Radulović, P, Balja, MP, Milošević, M. E-cadherin and NEDD9 expression in primary colorectal cancer metastatic lymph nodes and liver metastases. Oncol Lett. 2019;17(3):2881-2889. doi: 10.3892/ol.2019.9917

11. Zhou SL, Zhou ZJ, Hu ZQ. Tumor-associated neutrophils recruit macrophages and t-regulatory cells to promote progression of hepatocellular carcinoma and resistance to sorafenib. Gastroenterology. 2016;150(7):1646-1658.e17. doi: $10.1053 /$ j.gastro.2016.02.040
12. Shindoh J, Andreou A, Aloia TA. Microvascular invasion does not predict long-term survival in hepatocellular carcinoma up to $2 \mathrm{~cm}$ : reappraisal of the staging system for solitary tumors. Ann Surg Oncol. 2013;20(4):1223-1229. doi: 10.1245/s10434-012-2739-y

13. Ince V, Carr BI, Bag HG, Koc C, Usta S, Ersan V, et al. Gamma glutamyl transpeptidase as a prognostic biomarker in hepatocellular cancer patients especially with $>5 \mathrm{~cm}$ tumors, treated by liver transplantation. Int J Biol Markers. 2020;35(2):91-95. doi: $10.1177 / 1724600820921869$

14. Kim J, Soteriades ES, Christophi CA, Kales SN. Cancer incidence and mortality in firefighters: a state-of-the-art review and meta-analysis. Asian Pac J Cancer Prev. 2019;20(11):3221-3231. doi: 10.31557/ APJCP.2019.20.11.3221

15. Segal K, Cohen G, Gobin YP, Marr BP, Brodie SE, Dunkel IJ, et al. Salvage/adjuvant brachytherapy after ophthalmic artery chemosurgery for intraocular retinoblastoma. Int J Radiat Oncol Biol Phys. 2013;87(3):517-523. doi: 10.1016/j. ijrobp.2013.06.2045

16. Adachi $\mathrm{Y}$, Taniguchi $\mathrm{H}$, Moriya $\mathrm{C}$, Igarashi $\mathrm{H}$, Saitoh A, Yamamoto $\mathrm{H}$, et al. Cancer stem cells in human gastrointestinal cancer. Cancer Sci. 2016;107(11):15561562. doi: 10.1111/cas.13069

17. Kim, D.W, Talati, C, Kim, R. Hepatocellular carcinoma (HCC): beyond sorafenib-chemotherapy. J Gastrointest Oncol. 2017;8(2):256-265. doi: 10.21037/jgo.2016.09.07

18. Chou YC, Lao IH, Hsieh PL, Su YY, Mak CW, Sun DP, et al. Gadoxetic acid-enhanced magnetic resonance imaging can predict the pathologic stage of solitary hepatocellular carcinoma. World J Gastroenterol. 2019;25(21):2636-2649. doi: 10.3748/wjg.v25.i21.2636

\section{Author`s Contribution:}

KL conceived the study, literature review, data analysis, drafting the manuscript.

YP and HT helped in design, data collection, article drafting \& critical revision.

HH takes the responsibility and is accountable for all aspects of the work in ensuring that questions related to the accuracy or integrity of any part of the work are appropriately investigated and resolved. 